\title{
Special Issue on Algorithms and Models for Dynamic Multiple Criteria Decision Making
}

\author{
Debora Di Caprio ${ }^{1, *(D)}$ and Francisco Javier Santos Arteaga $2, *$ (D) \\ 1 Department of Economics and Management, University of Trento, 38122 Trento, Italy \\ 2 Faculty of Economics and Management, Free University of Bolzano, 39100 Bolzano, Italy \\ * Correspondence: debora.dicaprio@unitn.it (D.D.C.); fransant@ucm.es (F.J.S.A.)
}

check for updates

Citation: Di Caprio, D.; Santos Arteaga, F.J. Special Issue on Algorithms and Models for Dynamic Multiple Criteria Decision Making. Algorithms 2021, 14, 233. https:// doi.org/10.3390/a14080233

Received: 4 August 2021

Accepted: 6 August 2021

Published: 8 August 2021

Publisher's Note: MDPI stays neutral with regard to jurisdictional claims in published maps and institutional affiliations.

Copyright: (C) 2021 by the authors. Licensee MDPI, Basel, Switzerland. This article is an open access article distributed under the terms and conditions of the Creative Commons Attribution (CC BY) license (https:// creativecommons.org/licenses/by/ $4.0 /)$.

\section{Introductory Remarks}

The current Special Issue contains six papers focused on Multiple Criteria Decision Making (MCDM) problems and the formal techniques applied to derive consistent rankings of them. The wide applicability of these techniques is reflected in the variety of scenarios analyzed, which range from logistic systems to aeronautic engineering, encompassing several institutional applications. The papers which constitute this issue include both developments of novel techniques, extending the range of applications of MCDM models, and the implementation of several established methods used to develop extended hybrid models.

The substantial increment in the applications of MCDM techniques observed in recent years illustrates both their malleability and acceptance as problem-solving methods when dealing with conflicting criteria. A simple Google Scholar search for MCDM, without focusing on any particular technique, delivers more than 85,000 papers in the last 40 years, with each individual method increasing this count considerably. For instance, the Analytic Hierarchy Process (AHP) solely accounts for more than 750,000 papers. Despite the impressive evolution of this research area, there is still ample room for additional improvements dealing with unexplored, though essential, research aspects.

When dealing with conflicting objectives, the design of specific algorithms and heuristic methods seems restricted to their implementation within multi-objective optimization problems, particularly when analyzing complex structures involving strategic relations across variables. The current Special Issue aims to bridge the existing gap between standard MCDM methods, such as TOPSIS, VIKOR, PROMETHEE and ELECTRE, as well as the dynamic and strategic interactions taking place across variables in many real-life settings.

An intuitive example regarding the preferred type of research is provided by the different dynamic extensions of Data Envelopment Analysis (DEA) introduced in the operational research literature. Aside from the inclusion of a temporal dimension across MCDM methods, any potential development in techniques, such as dynamic DEA or novel extensions of multi-objective optimization problems with strategic interrelations across variables, were welcome as contributions to the current Special Issue.

\section{Special Issue}

The current special issue of Algorithms was designed to encourage the submission of original research on MCDM techniques and their potential applications. The call-for-papers invited articles which detailed the foundations of MCDM problems and their applications, with particular emphasis placed on strategic evaluation environments. Each of the articles submitted to the Special Issue was reviewed by at least two independent experts, whose detailed comments allowed authors to improve the quality of their presentations to a substantial extent.

The papers published can be divided into three main categories. The first category focuses on technical modifications and implementations of MCDM models. 
For instance, the paper by Tekile et al. [1] detailed the incompleteness in the pairwise comparison matrices which define Saaty's AHP. The authors proposed an extension of the Nelder-Mead simplex search algorithm to complete the matrix optimally by minimizing a maximum eigenvalue function subject to interval constraints and illustrating its applicability through several numerical simulations. Given the problems of data incompleteness faced in many real-life settings, this result constituted an important development that allowed for the implementation of AHP in scenarios that have generally remained out of its scope.

Di Caprio et al. [2] analyzed the design of unmanned vehicles equipped with deployable wings for suborbital flight. These vehicles were endowed with enhanced maneuverability and controlled landing features but required increasing levels of design complexity and faced housing difficulties in operative launchers. The authors developed a multi-objective optimization methodology that implemented a genetic algorithm together with a set of parametric models designed to identify the best configuration that minimized interface loads, improving the efficiency of the deployment and configuration systems, while increasing the payload and reducing service costs.

The second category of papers developed extensions of existing MCDM techniques, applied to analyze strategic decision environments.

The assignment of weights to the decision criteria, or experts providing their judgments constituted one of the most discussed topics in MCDM problems, particularly when dealing with group decision-making scenarios. Putro et al. [3] analyzed an extensive study case based on the design of logistic policies that supported sustainable mobility in the protected natural-cultural tourism area of Dieng in Central Java, Indonesia. These authors introduced a multi-actor multi-criteria analysis method where stakeholders were asked to select their own evaluation criteria and assess the involvement of other stakeholders. They analyzed and validated the consistency of the suggested procedure through AHP.

A similar case study in terms of consistency and applicability was developed by Moreira et al. [4], who defined a hybrid model based on PROMETHEE and SAPEVOM (Simple Aggregation of Preferences Expressed by Ordinal Method Vectors-Multi Decision Makers). Operating as a hybrid model, PROMETHEE-SAPEVO-M1 provided an integrated framework allowing for the ranking of environments dealing with quantitative and qualitative criteria, whose inputs were defined through cardinal and ordinal variables, respectively. The capacity of the model to deal with inaccurate information was illustrated via a case study formalizing the selection of attack helicopters for the Brazilian Navy.

The third and final category of papers corresponded to the implementation of existing MCDM models within extensive institutional case studies.

Oliveira et al. [5] ranked six business projects according to their potential capacity to be incubated. They applied the Momentum method to define an initial core of evaluation criteria. Then, the authors implemented the hybrid AHP-TOPSIS-2N technique to rank the projects, where AHP was used to derive the weights of each criterion while the decision matrix, representing the score assigned to each alternative per criterion, was normalized through two different standardization procedures.

Finally, Vavrek et al. [6] assessed the efficiency in the management of a homogenous group of 32 Czech municipalities using four key indicators combined into 15 groups, all of which were evaluated via TOPSIS. The authors verified statistically the results obtained through the Jaccard index. The study highlighted the importance that selecting suitable alternatives and indicators has for the outcomes obtained from a given MCDM model. This was achieved by deriving the combination of indicators that best portray the actual situation of the municipalities according to the opinions of the experts.

Author Contributions: Writing-original draft preparation, D.D.C. and F.J.S.A. All authors have read and agreed to the published version of the manuscript.

Funding: This research received no external funding.

Institutional Review Board Statement: Not applicable. 
Informed Consent Statement: Not applicable.

Data Availability Statement: Not applicable.

Acknowledgments: Debora Di Caprio and Francisco Javier Santos Arteaga, Guest Editors of this Special Issue, would like to thank the scholars for the high quality of the manuscripts submitted and the expert reviewers for their timely and highly detailed reviews. Finally, we would like to thank Alina Chen and the editorial office of the journal for their efficient assistance in the publishing process.

Conflicts of Interest: The authors declare no conflict of interest.

\section{References}

1. Tekile, H.; Fedrizzi, M.; Brunelli, M. Constrained eigenvalue minimization of incomplete pairwise comparison matrices by nelder-mead algorithm. Algorithms 2021, 14, 222. [CrossRef]

2. Di Caprio, F.; Scigliano, R.; Fauci, R.; Tescione, D. Design optimization of interfacing attachments for the deployable wing of an unmanned re-entry vehicle. Algorithms 2021, 14, 141. [CrossRef]

3. Putro, H.; Pradono, P.; Setiawan, T. Development of multi-actor multi-criteria analysis based on the weight of stakeholder involvement in the assessment of natural-cultural tourism area transportation policies. Algorithms 2021, 14, 217. [CrossRef]

4. Moreira, M.; Costa, I.D.A.; Pereira, M.; dos Santos, M.; Gomes, C.; Muradas, F. PROMETHEE-SAPEVO-M1 a hybrid approach based on ordinal and cardinal inputs: Multi-criteria evaluation of helicopters to support Brazilian navy operations. Algorithms 2021, 14, 140. [CrossRef]

5. Oliveira, A.; Gomes, C.; Clarkson, C.; Sanseverino, A.; Barcelos, M.; Costa, I.; Santos, M. Multiple criteria decision making and prospective scenarios model for selection of companies to be incubated. Algorithms 2021, 14, 111. [CrossRef]

6. Vavrek, R.; Bečica, J.; Papcunová, V.; Gundová, P.; Mitríková, J. Number of financial indicators as a factor of multi-criteria analysis via the TOPSIS technique: A municipal case study. Algorithms 2021, 14, 64. [CrossRef] 